OPEN ACCESS

Edited by:

Ravindra Deshpande Wake Forest School of Medicine,

United States

Reviewed by:

Gargi Mahapatra,

Wake Forest Baptist Medical Center,

United States

Angela Verma

University of Maryland, Baltimore,

United States

Praveen Kumar Allu,

University of Pennsylvania,

United States

*Correspondence:

Li-Wei Chen

leiwei@cgmh.org.tw

Specialty section

This article was submitted to

Gastroenterology,

a section of the journal

Frontiers in Medicine

Received: 28 August 2021

Accepted: 27 October 2021

Published: 22 November 2021

Citation:

Chen L-W, Chang L-C, Hua C-C,

Cheng T-C and Lee C-C (2021)

Comparing the Expressions of Vitamin

D Receptor, Cell Proliferation, and

Apoptosis in Gastric Mucosa With

Gastritis, Intestinal Metaplasia, or

Adenocarcinoma Change.

Front. Med. 8:766061.

doi: 10.3389/fmed.2021.766061

\section{Comparing the Expressions of Vitamin D Receptor, Cell Proliferation, and Apoptosis in Gastric Mucosa With Gastritis, Intestinal Metaplasia, or Adenocarcinoma Change}

\author{
Li-Wei Chen ${ }^{1,2 *}$, Liang-Che Chang ${ }^{3}$, Chung-Ching Hua ${ }^{4}$, Tzu-Chien Cheng ${ }^{3}$ and \\ Chin-Chan Lee ${ }^{2,4}$
}

${ }^{1}$ Department of Gastroenterology and Hepatology, Chang-Gung Memorial Hospital and University at Keelung, Keelung, Taiwan, ${ }^{2}$ Community Medicine Research Center, Chang-Gung Memorial Hospital and University at Keelung, Keelung, Taiwan, ${ }^{3}$ Department of Pathology, Pathology Chang-Gung Memorial Hospital and University at Keelung, Keelung, Taiwan, ${ }^{4}$ Department of Internal Medicine, Internal Medicine Chang-Gung Memorial Hospital and University at Keelung, Keelung, Taiwan

Background: This study aimed to compare the expression of vitamin D receptor (VDR), cell proliferation, and apoptosis in the gastric mucosa of patients with gastritis, intestinal metaplasia (IM), and adenocarcinoma using artificial intelligence.

Material and Methods: This study retrospectively enrolled patients at the Keelung Chang Gung Memorial Hospital from November of 2016 to June, 2017, who were diagnosed with gastric adenocarcinoma. The inclusion criteria were patients' pathologic reports that revealed all compartments of Helicobacter pylori infection, gastritis, IM, and adenocarcinoma simultaneously in the same gastric sample. Tissue slides after immunohistochemical $(\mathrm{IHC})$ staining were transformed into digital images using a scanner and counted using computer software (QuPath and ImageJ). IHC staining included PA1-711 antibody for VDR, Ki67 antigen for proliferation, and M30 antibody CK18 for apoptosis.

Results: Twenty-nine patients were included in the $\mathrm{HC}$ staining quantitative analysis. The mean age was $69.1 \pm 11.3 \mathrm{y} / \mathrm{o}$. Most (25/29, 86.2\%) patients had poorly differentiated adenocarcinoma. The mean expression of Ki67 and CK18 increased progressively from gastritis and IM to adenocarcinoma, with statistical significance $(P$ $<0.05)$. VDR expression did not correlate with Ki67 or CK18 expression. Survival time was only correlated with tumor stage (correlation coefficient $=-0.423, P$ value $<0.05$ ), but was not correlated with the expression of VDR, Ki67, and CK18.

Conclusion: Ki67 expression and CK18 expression progressively increased in the areas of gastritis, IM, and adenocarcinoma. No correlation between VDR expression and Ki67 or CK18 expression was found in this study.

Keywords: adenocarcinoma, stomach neoplasm, cell proliferation, apoptosis, artificial intelligence, Ki67 antigen, keratin 18 , receptor calcitriol 


\section{INTRODUCTION}

Gastritis and intestinal metaplasia (IM) are common findings in patients with Helicobacter pylori $(H$. pylori) infection $(1,2)$. The prevalence of IM in patients with $H$. pylori infection is $30-40 \%$ at the age of 50 years old $(1,2)$. Gastric epithelial hyperproliferation has been observed in patients with gastritis and IM caused by $H$. pylori infection (3-5). Previous studies have also revealed that IM is associated with an increased risk of gastric cancer (6-9). Detection of IM in gastric adenocarcinoma samples is a common histological finding (9).

Apoptotic cells are rare in the glandular neck region (the generative cell zone) of normal gastric mucosa. With progression of atrophic gastritis, the generative cell zone shifts downward and a relatively large number of apoptotic cells occur (10). H. pylori infection induces apoptosis in gastric epithelial cells (10). The effect of $H$. pylori apoptosis could result from molecules produced by $H$. pylori or the host immune/inflammatory response (10). Molecules such as cytotoxin (VacA), lipopolysaccharide, or nitric oxide may directly induce apoptosis $(10,11)$. Many cytokines produced by type $1 \mathrm{~T}$ helper cells, such as TNF- $\alpha$ and IFN- $\gamma$, markedly potentiate apoptosis. The balance between cell proliferation and apoptosis is important for carcinogenesis in precancerous lesions, such as IM and $H$. pylori infection $(10,11)$.

Gut epithelial vitamin D receptor (VDR) signaling appears to play an essential role in controlling mucosal inflammation and thus could be a useful therapeutic target in the management of some gastrointestinal diseases (12). Although 1, 25Dihydroxyvitamin D $\left[1,25(\mathrm{OH})_{2} \mathrm{D} 3\right]$ is not produced by the stomach, it affects the immune regulatory responses via the VDR of the stomach (13-15). Vitamin D deficiency has been associated with risk of several cancers including gastric cancer (16). VDR is a superfamily of steroid hormone receptors that act as a transcription factor for a target gene. Moreover, 1, 25 $(\mathrm{OH})_{2} \mathrm{D} 3$ was reported to be associated with the inhibition of cell cycle progression, induction of cell apoptosis, and differentiation of various types of cancer cells. Hence, 1, $25(\mathrm{OH})_{2} \mathrm{D} 3$ has been reported to inhibit proliferation and anti-tumor effects via VDR $(15,16)$. VDR was also reported to play an important role in gastric mucosa homeostasis and host protection against $H$. pylori infection (13). VDR could regulate cathelicidin antimicrobial protein (CAMP) and has an antimicrobial activity against $H$. pylori $(13,17)$. Past studies found an important role of the VDR/CAMP pathway in innate immunity and an antiinflammatory mechanism of vitamin D. VDR mRNA expression levels were significantly up-regulated in $H$. pylori-infected patients and positively correlated with chronic inflammation scores. There was a significant positive correlation between VDR and CAMP mRNA expression in H. pylori-positive gastric mucosa (13). In animal model study using wild-type and VDR knockdown mice to demonstrate that VitD3 inhibits $H$. pylori infection by enhancing the expression of VDR and CAMP. VDR knockdown mice were more susceptible to $H$. pylori infection. In cultured mouse primary gastric epithelial cells, VitD3/VDR complex binds to the CAMP promoter region to increase its expression (17).
1, $25(\mathrm{OH}) 2 \mathrm{D} 3$ binding to VDR could transcriptionally activate the expression of a number of target genes, finally executing the antitumor functions. A lot of genes have been identified as its direct targets, such as p21 $(18,19)$ and c-Myc $(20)$ which are involved in different signaling pathways during tumor genesis. A previous study has shown that vitamin D suppresses proliferation and stimulates cell cycle arrest in gastric cancer cells but not in immortalized normal gastric cells (12). Vitamin D has increased p21 expression and decreased cyclin-dependent kinase 2 (CDK2) expression via VDR route (12).

The hypothesis of this study was that patients infected with $H$. pylori might have gastritis, gastric IM, and gastric adenocarcinoma. VDR expression in the stomach may differ in the areas of gastritis, IM, and gastric adenocarcinoma. When compared with the area of gastritis, the pathologic presentation of cell proliferation and apoptosis may be different in the areas of IM and adenocarcinoma. A progressive increase or decrease in cell proliferation or apoptosis may be detected in the areas of gastritis, IM, and gastric cancer, respectively.

The current study aimed to evaluate VDR expression, cell proliferation, and apoptosis in gastric adenocarcinoma samples using digital quantitative immunohistochemistry (IHC) statin analyses. In this study we aimed to:

1. Compare the Expression of VDR, Cell Proliferation (by Ki67 Statin), and Apoptosis (by CK18 Staining) in three Different Parts, Including Gastritis (non-Dysplasia or Tumor), IM (Premalignant Area), and Gastric Adenocarcinoma (Malignant Site) by Artificial Intelligence (AI) Using Computer Software to Prevent Manual or Inter-Observer Bias for IHC Score Counting;

2. Analyze the Association Between VDR Expression, Cell Proliferation, and Apoptosis;

3. Elucidate Whether High VDR Expression Is Associated With Higher or Lower Cell Proliferation and Apoptosis;

4. Analyze the Correlation of Survival Time With Tumor Stage and three IHC Stain Expressions.

\section{MATERIALS AND METHODS}

\section{Subjects and Tissue Samples}

This study retrospectively enrolled patients who were diagnosed with gastric adenocarcinoma at the Keelung Chang Gung Memorial Hospital (KCGMH) from November of 2016 to June 2017. Gastric samples that were confirmed as malignant after pathologic examination were stored in the tissue bank of KCGMH. For a patient with both endoscopic biopsy and surgical resection specimens, samples from surgical resection were used for IHC staining expression analyses in this study. For patients without an operation for gastric malignancy, endoscopic biopsy samples were used for IHC analyses. The inclusion criteria were patients' pathologic reports that revealed all compartments of $H$. pylori infection, gastritis, IM, and adenocarcinoma simultaneously in the same gastric sample. The exclusion criteria were incomplete records of demographic data, tumor stage, and clinical course. 
Patient demographics, tumor location in the stomach, tumor stage, and survival time were recorded. No chemotherapy, radiotherapy, or other therapies were performed in these patients before endoscopic biopsy or surgical resection. Two pathologists (Dr. Chang LC and Dr. Cheng TC) provided all the equipment and histological examinations. Every specimen was reviewed by an experienced pathologist (Dr. Chang LC) under microscopic examination to localize the areas of gastritis, IM, and adenocarcinoma. Commercial kits for IHC staining and publicly available software applications for digital image creation and quantitative analysis were used. This study was approved by the Ethics Committee of the Chang Gung Memorial Hospital (IRB No 103-7463A3, 105-4426C).

\section{Histology and IHC Stain for H. pylori Detection and IM}

Histology (hematoxylin and eosin) and IHC H. pylori antibody staining (polyclone, Zytomed Systems GmbH, Berlin, Germany) were performed to confirm $H$. pylori infection. Histological sections of all specimens were routinely examined to determine gastritis, IM, and malignancy. Because the gastric mucosa adjacent to malignancy is always infiltrated by inflammatory cell, a diagnosis of gastritis is made for this non-tumor, nonIM mucosa by pathologists. IM was detected based on the morphological features in the stomach observed by H\&E and Alcian blue staining $(6,21)$.

\section{Digital Quantitative Analyses for IHC Images}

Tissue sections were cut from the tissue blocks at $4 \mu \mathrm{m}$ and stained with $\mathrm{H} \& \mathrm{E}$ and IHC. The slides were scanned at $400 \times$ magnification using a Hamamatsu Nanozoomer S360 scanner and NDP image (Hamamatsu, Japan). Digital data analysis was performed using computer software to prevent manual or inter-observer bias for IHC score counting. The computer softwares ImmunoRatio (ImageJ plugin) (22) and QuPath (23) were applied for digital slide bioimage analyses (24-26). The application of ImmunoRatio calculates the percentage of positively stained nuclear area (labeling index) by using a color deconvolution algorithm for separating the staining components (diaminobenzidine and hematoxylin) and adaptive thresholding for nuclear area segmentation (25). Every digital image was quantitatively analyzed in three adjacent areas: gastritis, IM, and adenocarcinoma.

\section{Ki-67 Antigen for Epithelial Cell Proliferation}

Antigen Ki-67 IHC staining was performed using a DAKO autostain agent (Cytomation, Carpinteria, CA, USA). The REAL EnVision Detection System, Peroxidase/diaminobenzidine (DAB) (K5007, DAKO), was used to visualize the staining.

\section{PA1-711 Antibody for VDR}

After appropriate blocking and management of gastric tissue, VDR staining was performed using an anti-VDR polyclonal antibody (PA1-711, Thermo Scientific, Fremont, CA, USA).

\section{M30 CytoDEATH Antibody for Detecting Apoptosis}

Mouse monoclonal antibody (Clone M30, mouse IgG2b) was used to detect apoptosis in epithelial cells (caspase cleavage product of cytokeratin 18, CK18). Apoptosis was detected by applying the M30-antibody to fixed samples, and then secondary detection systems were used for IHC staining. The M30 CytoDEATH antibody (Roche Diagnostics GmbH, Mannheim, Germany) binds to a caspase-cleaved, formalin-resistant epitope of the CK 18 cytoskeletal protein (27).

\section{Gastric Cancer Stage}

Gastric cancer staging was performed according to the American Joint Committee on Cancer (AJCC, 7th edition) (28).

\section{Statistical Analysis}

Continuous data are expressed as mean \pm standard deviation (SD). A two-sample $t$-test was used to compare the mean values. Categorical data were analyzed using chi-square and Fisher exact tests. One-way analysis of variance (ANOVA) was used to compare the mean values of multiple samples. The Scheffe method was applied for post-hoc analysis. All statistical tests were two-tailed. Differences were considered statistically significant at $p<0.05$. Statistical analyses were performed using the Statistical Package for the Social Sciences (version 18.0) for Windows (PASW, Chicago, IL, USA).

\section{RESULTS}

Initially, 69 gastric tissue samples were collected from the tissue bank of KCGMH. Among these 69 samples, 32 originated from the same patients (from endoscopic biopsy and surgical resection). Five samples were inadequate for IHC staining examination. We were unable to trace the origins of three samples. Finally, 29 patients (24 surgical resections and 5 endoscopic biopsies) were included in the IHC staining analysis (Figure 1, Study flow). IHC stains of PAI 711 antibody (VDR), Ki67 antigen (cell proliferation), and M30 antibody CK18 (cell apoptosis) were performed for all samples from these 29 patients.

The demographic and clinical characteristics of the patients are listed in Table 1. The mean age was $69.1 \pm 11.3$ y/o. Most $(25 / 29,86.2 \%)$ patients had poorly differentiated adenocarcinoma. The tumors were located in the gastric antrum $(22 / 29,75.9 \%)$, body $(6 / 29,20.7 \%)$, and cardiac $(1 / 29,3.4 \%)$. Tumor stages at diagnosis were stage I $(13 / 29,44.8 \%)$, stage II $(2 / 29,6.9 \%)$, stage III $(6 / 29,20.7 \%)$, and stage IV $(8 / 29,27.6 \%)$.

Digital images of $H$. pylori infection ( $\mathrm{H} \& \mathrm{E}$ and antibody stain, Figure 2). Figures 3, 4 reveals digital images of $\mathrm{H} \& \mathrm{E}$ and CK18 stain in different sites (total, gastritis, IM, and adenocarcinoma). Digital analyses of IHC expression using ImageJ and ImmunoRatio software are presented in Figure 5. Figure 6 reveals different cell types (moderate differentiation and well-differentiation) gastric adenocarcinoma and IHC (VDR, Ki67 and CK18) stain.

The expression levels of PAI 711 antibody (VDR), Ki67 antigen (cell proliferation), and M30 antibody CK18 (cell apoptosis) among gastritis, IM, and adenocarcinoma are listed 


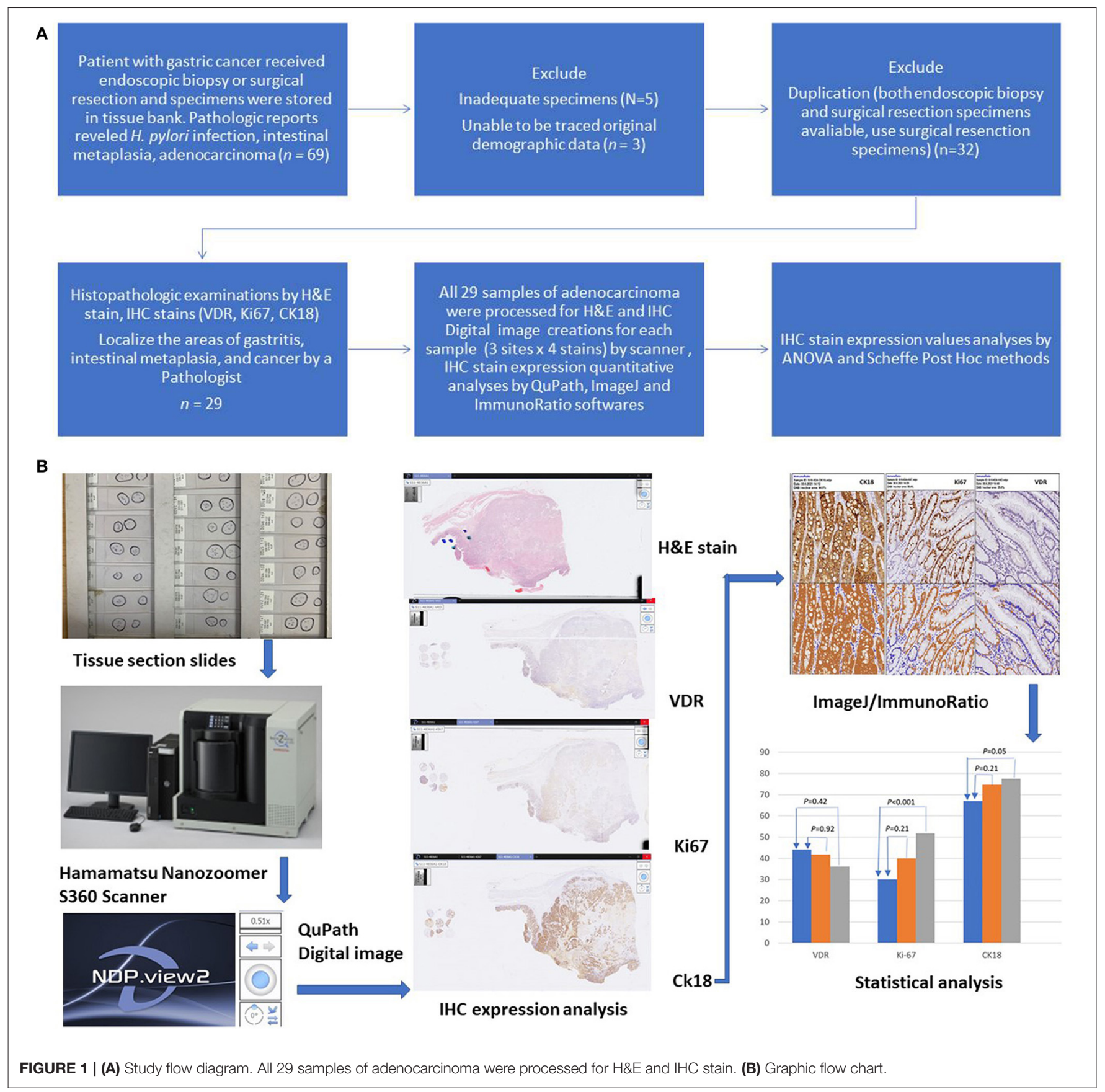

in Table 2. The mean expression of Ki67 and CK18 increased significantly $(P<0.05)$ from gastritis, IM to adenocarcinoma. However, mean values of VDR expression were no statistical difference among gastritis, IM, and adenocarcinoma by ANOVA $(P=0.404)$.

Table 3 shows the Scheffe post-hoc analysis. The main location for a different expression of Ki67 and CK18 were between gastritis and cancer. The expression of Ki67 and CK18 was similar between the IM (premalignancy) and adenocarcinoma (malignancy) sites (Figure 7).
When a correlation analysis between VDR and Ki67 or CK18 was performed, VDR expression was not correlated with Ki67 or CK18 expression in gastritis, IM and adenocarcinoma site (Table 4).

Compare the VDR expression in gastritis, IM, and adenocarcinoma between male and female patients. There was no statistical difference of mean VDR expression between male and female patients in the gastritis ( $42.2 \pm 25.0$ vs. $48.9 \pm$ 27.7, $P=0.58)$, IM ( $41.8 \pm 17.3$ vs. $41.7 \pm 14.7, P=0.73)$, and adenocarcinoma $(36.6 \pm 18.8$ vs. $36.6 \pm 18.5, P=0.98)$. 
Survival time was only correlated with tumor stage (correlation coefficient $=-0.423, P$-value $<0.05$ ), but was not correlated with the expression of VDR, Ki67, and CK18.

\section{DISCUSSION}

VDR could regulate cathelicidin antimicrobial protein (CAMP) and has an antimicrobial activity against $H$. pylori $(13,17)$. Past studies found an important role of the VDR/CAMP pathway in innate immunity and an anti-inflammatory mechanism of vitamin D $(13,17)$. VDR mRNA expression levels were significantly up-regulated in $H$. pylori-infected patients and positively correlated with chronic inflammation scores. There was a significant positive correlation between VDR and CAMP mRNA expression in H. pylori-positive gastric mucosa (13).

TABLE 1 | Demography and clinical characters.

\section{Age $(\mathrm{y} / \mathrm{o})$}

Gender (F/M)

Adenocarcinoma type (tubular/other)

Tumor location (antrum/body/cardiac)

Differentiation (poor/moderate/well)

Operation (with/without) after diagnosis

Operation type (BI/BII/Total gastrectomy)

Tumor stage (I/II/III/IV)

Chemotherapy (with/without) after diagnosis

Mean survival days (stage I/II/III/IV)

IM, intestinal metaplasia; BI, Billroth's I subtotal gastrectomy; Bll, Billroth's II subtotal gastrectomy; Total, total gastrectomy.
Previous studies have shown that VDR expression may control the expression of cell proliferation and apoptosis (14-16, 29-31). Wen et al. (32) demonstrated that VDR was lowest in cancer tissues (positive rate $=57.61 \%$ ), compared to premalignant (73.64\%) and normal tissues (82.61\%), with a statistically significant difference $(P=0.001)$. A decline in VDR expression was observed in normal, premalignant, and malignant gastric tissues, especially in poorly differentiated tissues. In our study,

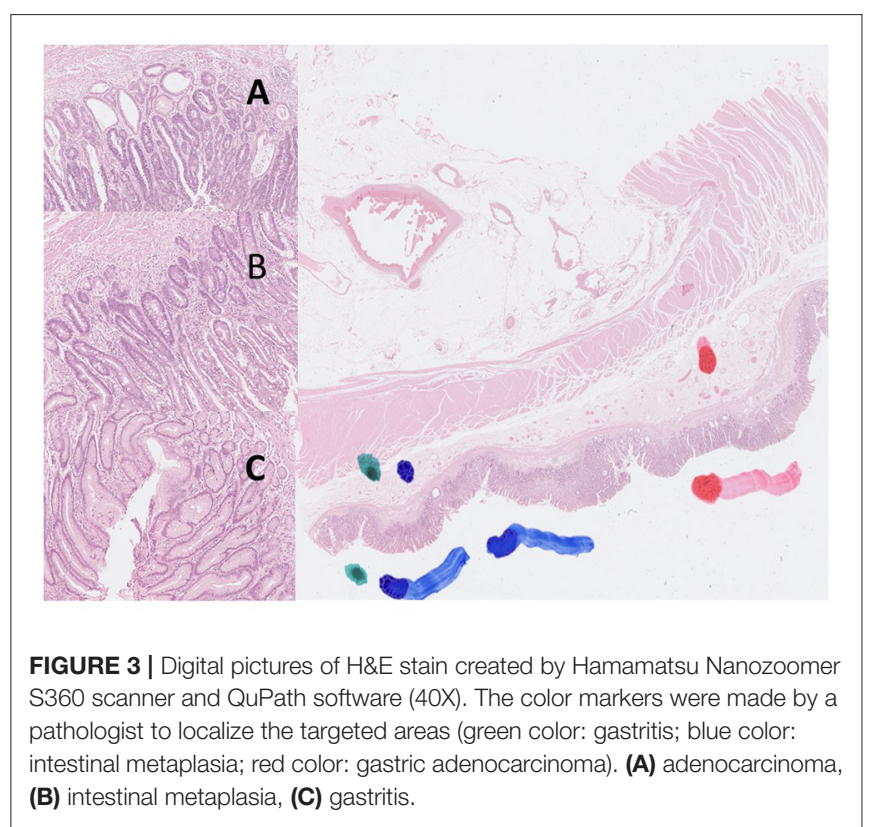

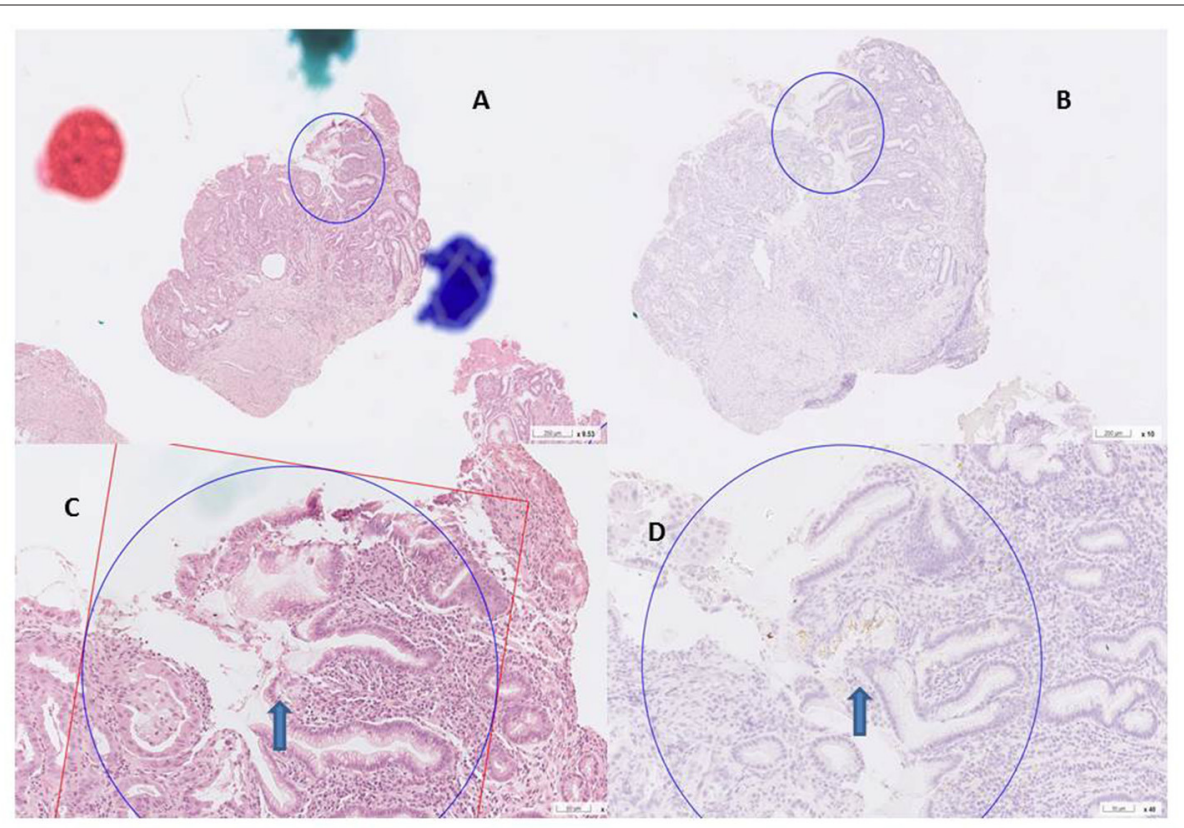

FIGURE $2 \mid$ H. pylori infection H\&E and antibody stain. (A,B) H\& E stain, curved bacilli within the mucus of epithelim (arrow). (C,D) anti H. pylori antibody stain (arrow). 
there was no statistically difference of VDR expression (44.1\%, $41.8 \%, 36.1 \%$ in gastritis, IM, and malignant tissue, respectively; $P=0.404$ ) was detected. The main reason for the difference in results between Wen et al. (32) and our study may be due to the different methods for VDR expression evaluation. In Wen et al. study, VDR expression was presented as a positive rate (PR), which was similar to qualitative analysis (positive or negative). Moreover, some gastritis or IM tissues were collected from patients without gastric cancer. In our study,

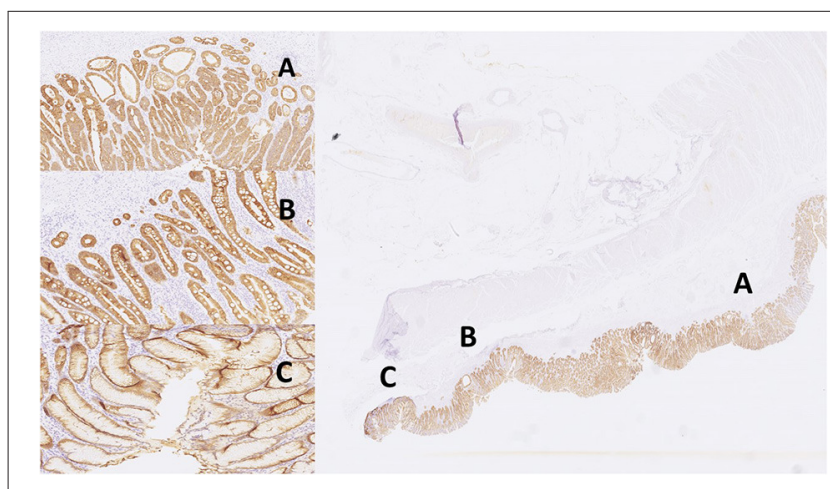

FIGURE 4 | Images of IHC expression by CK18 stain in different sites. Right: total; left top: gastritis; left middle: IM; left bottom: adenocarcinoma. (A) adenocarcinoma, (B) intestinal metaplasia, (C) gastritis. quantitative digital analyses were performed to prevent intra-or inter-observer bios. All three parts (gastritis, IM, and cancer) were adjacent and came from the same patient. Because the major type of gastric adenocarcinoma was poorly differentiated in this study, similar VDR expression in the mucosa adjacent to the tumor may be found in patients with poorly differentiated gastric adenocarcinoma.

The relationship between serum vitamin D deficiency and gastric cancer development remains under debate $(13-15,33,34)$. Because $1,25(\mathrm{OH})_{2} \mathrm{D} 3$ is not produced by the stomach, 1,25 $(\mathrm{OH})_{2} \mathrm{D} 3$ affects the immune regulatory responses via the VDR of the stomach (12-14). However, no serum vitamin D level was recorded in this study because checking the vitamin D level was not a regular guideline for gastric adenocarcinoma treatment. To understand the relationship between serum vitamin D level and gastric VDR expression, it is necessary to include more patients with serum vitamin D and VDR expression in the gastric mucosa by endoscopic biopsy.

It is common to disclose increased proliferation and decreased apoptosis in malignant cells (10). Our hypothesis was an increased Ki67 (cell proliferation) and a deceased CK18 (apoptosis) in cancer, but the results of our study revealed that both Ki67 and CK18 expression increased in IM and malignant tissues. Apoptosis is defined by characteristic changes in nuclear morphology, including chromatin condensation and fragmentation, overall cell shrinkage, and blebbing of the plasma membrane. CK18 expression was high (mean 77.6\%) in gastric
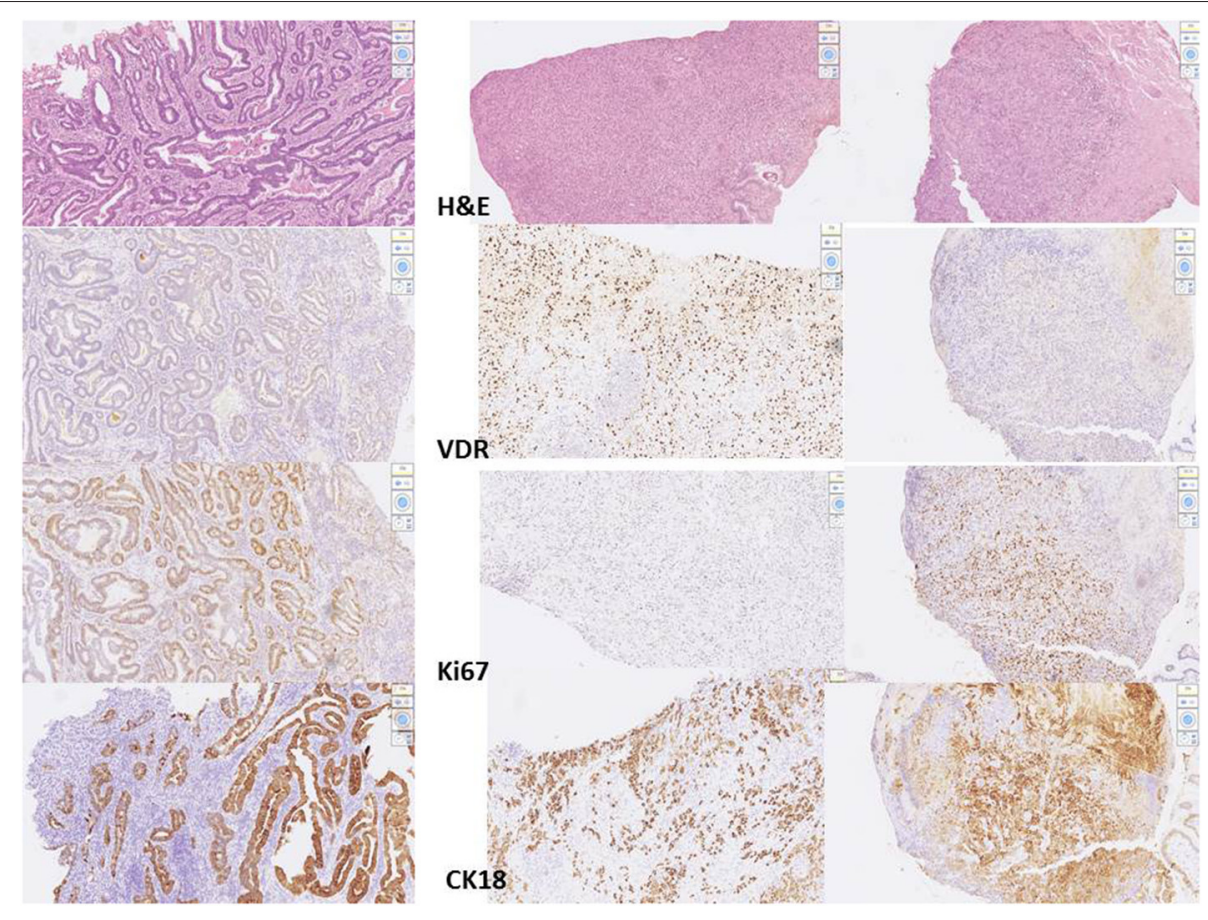

FIGURE 5 | Digital images for different cell type of adenocarcinoma from 3 patients. Left: moderate differentiated adenocarcinoma, stage III. Middle: poor differentiated adenocarcinoma, stage I. Right: poor differentiated adenocarcinoma, stage III. From the top to the bottom: H\&E stain, VDR, Ki67, CK18 stain processed by scanner and QuPath software. 


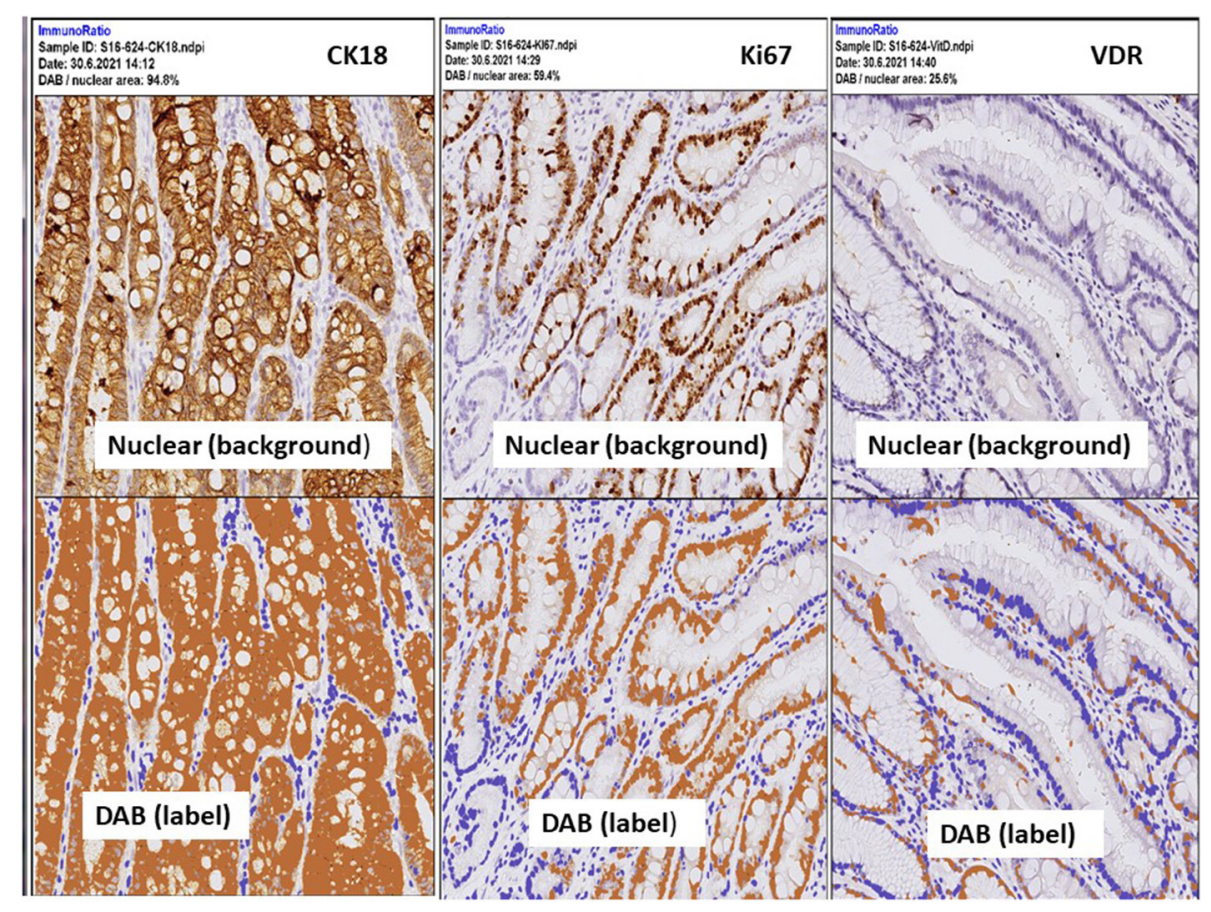

FIGURE 6 | IHC expressions by ImmunoRatio (ImageJ plugin software) processed from one patient with stage I, poor differentiated type gastric adenocarcinoma. Upper panel: the original hematoxylin image (total cell). Lower panel: "DAB" indicates labeled cells, a pseudo-colored image showing the segmented staining components. Labeling index is calculated by the percentage of DAB (positively stained cells) divided the total cell. Left: $94.8 \%$ by CK18, middle: $59.4 \%$ by Ki67, right: $25.8 \%$ by VDR.

TABLE 2 | The mean expressions of VDR, Ki67, and CK18 among gastritis, IM and cancer.

\begin{tabular}{lcccr}
\hline & \multicolumn{4}{c}{ Mean expression (\%) in targeted areas } \\
\cline { 2 - 5 } IHC stain & Gastritis & IM & Cancer & P-value \\
\hline VDR & $44.1 \pm 26.4$ & $41.8 \pm 16.9$ & $36.1 \pm 19.7$ & 0.404 \\
Ki67 & $30.1 \pm 18.4$ & $39.9 \pm 18.5$ & $51.8 \pm 21.8$ & $<0.001$ \\
CK18 & $66.9 \pm 19.8$ & $74.7 \pm 15.0$ & $77.6 \pm 14.7$ & 0.047 \\
\hline
\end{tabular}

IHC stain, immunohistochemical stain; Gastritis, inflammation but relatively normal gastric mucosa adjacent to malignancy; IM, intestinal metaplasia adjacent to malignancy; Cancer, gastric adenocarcinoma; VDR, PA1-711 for vitamin D receptor; Ki67, antigen Ki-67 stain for epithelial cell proliferation; CK18, cytokeratin 18, M30 CytoDEATH antibody for detecting apoptosis; P-value, ANOVA analysis.

adenocarcinoma in the current study. The expression of CK18 was also high in the non-tumor gastritis mucosa in the current study (mean 66.9\%).

Chronic inflammation and IM are associated with increased apoptosis but primarily occur at the mucosal surface and not in the deeper layers (27). In the current study, the mucosa of gastritis was adjacent to the IM and the malignant areas. Chronic inflammation may induce high CK18 expression (35). Previous studies on apoptosis in gastric adenocarcinoma mostly used cell lines and the counting score for apoptosis was variable $(10,35)$. CK18 expression in the mucosa of gastritis adjacent to
TABLE 3 | Scheffe post hoc analysis among different stains and locations.

\begin{tabular}{|c|c|c|c|c|c|c|}
\hline \multirow{2}{*}{$\begin{array}{l}\text { Stain } \\
\text { Location }\end{array}$} & \multicolumn{2}{|c|}{ VDR } & \multicolumn{2}{|c|}{ Ki67 } & \multicolumn{2}{|c|}{ CK18 } \\
\hline & Gastritis & IM & Gastritis & IM & Gastritis & IM \\
\hline \multicolumn{7}{|c|}{ Adenocarcinoma+ } \\
\hline Coefficient & -7.362 & -5.103 & 21.128 & 11.931 & 10.638 & 2.851 \\
\hline$P$-value & 0.421 & 0.659 & 0.000 & 0.069 & 0.057 & 0.809 \\
\hline
\end{tabular}

+ Post hoc compare the expression of IHC between adenocarcinoma and gastritis or intestinal metaplasia.

IM and gastric adenocarcinoma (especially poor differentiation type) may be high in nature. An imbalance between cell proliferation and apoptosis may be the reason and mechanism of carcinogenesis $(10,17)$. Wagner et al. (29) found decreased apoptotic activity following increased proliferation in chronic $H$. pylori infection; they assumed that increased proliferation might play a role in carcinogenesis.

In normal gastric mucosa, apoptotic cells are rare and in the generative cell zone near glandular neck region. When the sequential change of atrophic gastritis, IM, and dysplasia, the number of apoptotic cells increase (10). Numerous molecules produced by $H$. pylori including cytotoxin (VacA), lipopolysaccharide, monochloramine, and nitric oxide may directly induce apoptosis. Moreover, H. pylori-stimulated host inflammatory/immune responses lead to release of a large 


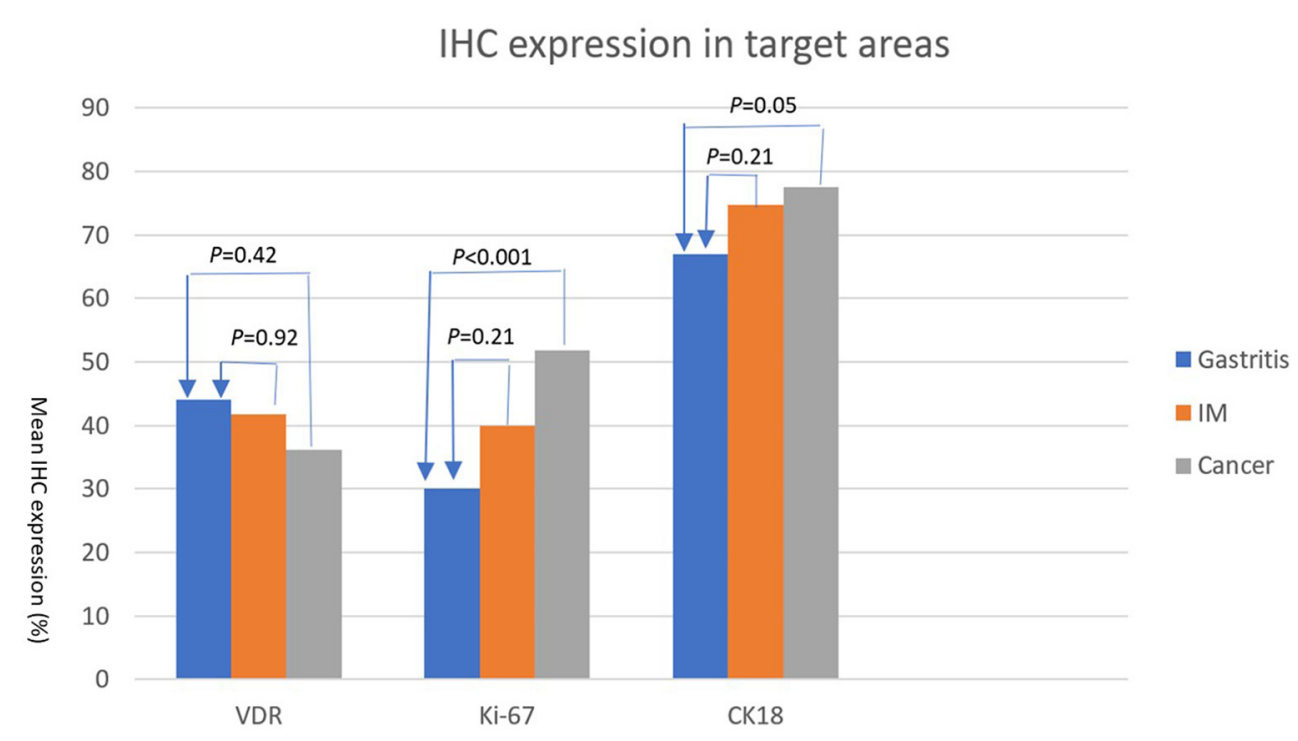

FIGURE 7 | IHC expressions (\%) $(Y$ axis) of VDR, Ki67, and CK18 (X axis) in areas of gastritis, IM, and cancer. The expression of each IHC stain in IM (orange bar) and cancer (gray bar) is compared with the expression in gastritis (blue bar). P-values are presented at the top of each comparison (Scheffe post-hoc analysis).

TABLE 4 | Correlation the expressions between VDR and Ki67 or CK18 in different sites.

\begin{tabular}{lccc}
\hline $\begin{array}{l}\text { Area } \\
\text { referred factor }\end{array}$ & Compared factor & $\begin{array}{c}\text { Correlation } \\
\text { coefficient }\end{array}$ & P-value \\
\hline $\begin{array}{l}\text { Gastritis } \\
\text { VDR }\end{array}$ & Ki67 & 0.142 & 0.462 \\
& CK18 & -0.121 & 0.531 \\
IM & Ki67 & 0.171 & 0.375 \\
VDR & CK18 & -0.302 & 0.111 \\
Cancer & Ki67 & 0.066 & 0.736 \\
VDR & CK18 & 0.103 & 0.597 \\
\hline
\end{tabular}

amount of cytokines. Cytokines produced by type $1 \mathrm{~T}$ helper cells, such as TNF-alpha and IFN-gamma, markedly potentiate apoptosis $(10,36)$.

This study applied artificial intelligence (AI) methods, including a high-resolution scanner (Hamamatsu Nanozoomer S360 scanner) for digital image creation, QuPath, and ImageJ/ImmunoRatio software for IHC scoring. This software could be downloaded freely from specific webs. The strength of AI use is rapid and avoids intra- or inter-observer bios. AI for IHC score counting is easier than traditional manual counting. The preparing of samples in this study were paraffin-embedded tissue sections from tissue bank. Paraffin-embedded tissues are frequently used for pathological examinations, included IHC analyses. Patients who get $H$. pylori infection may develop the conditions of gastritis, IM and gastric adenocarcinoma, which conditions could be detected in one slicing slide from the surgical or biopsy specimens. IHC examination could evaluate the expressions of VDR, Ki67 and CK18 with precise locations in one slide simultaneously. However, there are some limitations to use AI for quantitative IHC tests like our study. First, the precisely targeted areas for scanning and scoring still depend on the pathologists in our study. Second, reports issued the correlation between IHC quantitative analyses and gene expressions in this field of $H$. pylori related gastric adenocarcinoma are rarely now. Third, the application of ImageJ/ImmunoRatio software for other different IHC examinations may require further studies. Hence, further studies are needed for validation and clarification the correlation between digital IHC quantitative tests and gene expression examination.

Conclusively, cell proliferation by $\mathrm{Ki67}$ expression and apoptosis by CK18 expression progressively increased in the areas of gastritis, IM, and adenocarcinoma. Ki67 expression positively correlated with CK18 expression in gastritis. No association between VDR expression and Ki67 or CK18 expression was found in this study. Survival time was only correlated with tumor stage, but was not correlated with the expression of VDR, Ki67, and CK18.

\section{DATA AVAILABILITY STATEMENT}

The original contributions presented in the study are included in the article, further inquiries can be directed to the corresponding author.

\section{ETHICS STATEMENT}

The Institutional Review Board of the Chang-Gung Memorial Hospital approved this research (IRB No. 103-7463A3, 105-4426C). All participants agreed to the study conditions 
and provided informed consent before the enrollment in this study.

\section{AUTHOR CONTRIBUTIONS}

L-WC, L-CC, and C-CH contributed to conception and design of the study. L-WC organized the database, wrote the first draft of the manuscript, and got the grant. L-WC, C-CH, and $\mathrm{C}-\mathrm{CL}$ performed the statistical analysis. L-WC and L-CC wrote sections of the manuscript. L-CC and T-CC provided all the equipment and histological examinations. All authors contributed to manuscript revision, read, and approved the submitted version.

\section{REFERENCES}

1. Wong BC, Lam SK, Wong WM, Chen JS, Zheng TT, Feng RE, et al. Helicobacter pylori eradication to prevent gastric cancer in a high-risk region of China: a randomized controlled trial. JAMA. (2004) 291:187-94. doi: 10.1001/jama.291.2.187

2. Asaka M, Sugiyama T, Nobuta A, Kato M, Takeda H, Graham DY. Atrophic gastritis and intestinal metaplasia in Japan: results of a large multicenter study. Helicobacter. (2001) 6:294-9. doi: 10.1046/j.1523-5378.2001.00042.x

3. Bechi P, Balzi M, Becciolini A, Maugeri A, Raggi CC, Amorosi A, et al. Helicobacter pylori and cell proliferation of the gastric mucosa: possible implications for gastric carcinogenesis. Am J Gastroenterol. (1996) 91:271-6.

4. Ierardi E, Francavilla R, Panella C. Effect of Helicobacter pylori eradication on intestinal metaplasia and gastric epithelium proliferation. Ital J Gastroenterol Hepatol. (1997) 29:470-5.

5. Peek RM Jr, Moss SF, Tham KT, Holt PR, Tham KT, Blaser MJ, et al. Helicobacter pylori cagA+ strains and dissociation of gastric epithelial cell proliferation from apoptosis. J Nat Cancer Inst. (1997) 89:863-8. doi: 10.1093/jnci/89.12.863

6. Correa P, Piazuelo B, Wilson KT. Pathology of gastric intestinal metaplasia: clinical implications. Am J Gastroenterol. (2010) 105:493-8. doi: 10.1038/ajg.2009.728

7. Sung JJY, Lin SR, Ching JYL, Zhou LY, To KF, Wang RT, et al. Atrophy and intestinal metaplasia one year after cure of $\mathrm{H}$ pylori infection: a prospective, randomized study. Gastroenterology. (2000) 119:714. doi: 10.1053 /gast.2000.8550

8. Leung WK, Lin SR, Ching JYL, To KF, Ng EK, Chan FK, et al. Factors predicting progression of gastric intestinal metaplasia: results of a randomised trial on Helicobacter pylori eradication. Gut. (2004) 53:1244-9. doi: 10.1136/gut.2003.034629

9. Zhang C, Yamada N, Wu YL, Wen M, Matsuhisa T, Matsukura N. Helicobacter pylori infection, glandular atrophy and intestinal metaplasia in superficial gastritis, gastric erosion, erosive gastritis, gastric ulcer and early gastric cancer. World J Gastroenterol. (2005) 11:791-6. doi: 10.3748/wjg.v11.i6.791

10. Xia HH, Talley NJ. Apoptosis in gastric epithelium induced by Helicobacter pylori infection: implications in gastric carcinogenesis. Am J Gastroenterol. (2001) 96:16-26. doi: 10.1016/S0002-9270(00)02 240-1

11. Yousefi B, Mohammadlou M, Abdollahi M, Salek Farrokhi A, Karbalaei M, Keikha M, et al. Epigenetic changes in gastric cancer induction by Helicobacter pylori. J Cell Physiol. (2019) 234:21770-84. doi: $10.1002 /$ jcp. 28925

12. Li M, Li L, Zhang L, Hu W, Shen J, Xiao Z, et al. 1,25-Dihydroxyvitamin D3 suppresses gastric cancer cell growth through VDR- and mutant p53-mediated induction of p21. Life Sci. (2017) 179:88-97. doi: 10.1016/j.lfs.2017.04.021

13. Guo L, Chen W, Zhu H, Chen Y, Wan X, Yang N, et al. Helicobacter pylori induces increased expression of the vitamin $\mathrm{d}$ receptor in immune responses. Helicobacter. (2013) 19:37-47. doi: 10.1111/hel.12102

\section{FUNDING}

This study was supported by the $\begin{array}{r}\text { Chang } \\ \text { Gung Medical } \\ \text { Gung Meundation and Keelung }\end{array}$ Chang
2H0052).

\section{ACKNOWLEDGMENTS}

The authors thank two assistants from the Community Medicine Research Center, Chia-Wen Hsieh and Mi-Sio Huang for assisting with sample preparation from the tissue bank and clinical data collection.

14. Ben C, Qiu WZ, Wang DS, Luo HY, Zhang DS, Wang ZQ, et al. Prognostic effects of 25-hydroxyvitamin D levels in gastric cancer. J Trans Med. (2012) 10:16-20. doi: 10.1186/1479-5876-10-16

15. Bao A, Li Y, Tong Y, Zheng H, Wu W, Wei C. Tumor-suppressive effects of 1,25-dihydroxyvitamin D3 in gastric cancer cells. Hepato Gastroenterol. (2013) 60:943-8. doi: 10.5754/hge121003

16. Du C, Yang S, Zhao X, Dong H. Pathogenic roles of alterations in vitamin D and vitamin D receptor in gastric tumorigenesis. Oncotarget. (2017) 8:2947486. doi: 10.18632/oncotarget.15298

17. Zhou A, Li L, Zhao G, Min L, Liu S, Zhu S, et al. Vitamin D3 inhibits helicobacter pylori infection by activating the VitD3/VDRCAMP pathway in mice. Front Cell Infect Microbiol. (2020) 10:566730. doi: 10.3389/fcimb.2020.566730

18. Saramaki A, Banwell CM, Campbell MJ, Carlberg C. Regulation of the human p21(waf1/cip1) gene promoter via multiple binding sites for p53 and the vitamin D3 receptor. Nucleic Acids Res. (2006) 34:543-54. doi: 10.1093/nar/gkj460

19. Rao A, Coan A, Welsh JE, Barclay WW, Koumenis C, Cramer SD. Vitamin D receptor and p21/WAF1 are targets of genistein and 1,25dihydroxyvitamin D3 in human prostate cancer cells. Cancer Res. (2004) 64:2143-7. doi: 10.1158/0008-5472.CAN-03-3480

20. Salehi-Tabar R, Nguyen-Yamamoto L, Tavera-Mendoza LE, Quail T, Dimitrov $\mathrm{V}$, An BS, et al. Vitamin D receptor as a master regulator of the cMYC/MXD1 network. Proc Natl Acad Sci USA. (2012) 109:18827-32. doi: 10.1073/pnas.1210037109

21. Capelle LG, de Vries AC, Haringsma J, Ter Borg F, de Vries RA, Bruno MJ, et al. The staging of gastritis with the OLGA system by using intestinal metaplasia as an accurate alternative for atrophic gastritis. Gastrointest Endosc. (2010) 71:1150-8. doi: 10.1016/j.gie.2009.12.029

22. ImmunoRatio 1.0c.Jar Downloaded. Available online at: https://wsiserver.jilab. fi/old-jvsmicroscope-software/ (accessed March 7, 2019).

23. QuPath version 0.23 Downloaded. Available online at: https://qupath.github. io/ (accessed September 27, 2020).

24. Schneider CA, Rasband WS, Eliceiri KW. NIH Image to ImageJ: 25 years of image analysis. Nat Meth. (2012) 9:671-5. doi: 10.1038/nmeth.2089

25. Tuominen VJ, Ruotoistenmäki S, Viitanen A, Jumppanen M, Isola J. ImmunoRatio: a publicly available web application for quantitative image analysis of estrogen receptor (ER), progresterone receptor (PR), and Ki-67. Breast Cancer Res. (2012) 12:R56. doi: 10.1186/bcr2615

26. Bankhead P, Loughrey MB, Fernández JA, Dombrowski Y, McArt DG, Dunne PD, et al. QuPath: open source software for digital pathology image analysis. Sci Rep. (2017) 7:16878. doi: 10.1038/s41598-017-172 04-5

27. Leers MPG, Lgen WK, Rklund VB, Bergman T, Tribbick G, Persson B, et al. Immunocytochemical detection and mapping of a cytokeratin 18 neo-epitope exposed during early apoptosis. J Pathol. (1999) 187:567-72. doi: 10.1002/ (SICI)1096-9896(199904)187:5<567::AID-PATH288>3.0.CO;2-J

28. Washington K. 7th edition of the AJCC cancer staging manual: stomach. Ann Surg Oncol. (2010) 17:3077-9. doi: 10.1245/s10434-010-1 $362-\mathrm{z}$ 
29. Wagner S, Beil W, Westermann J, Logan RPH, Bock CT, Trautwein C, et al. Regulation of gastric epithelial cell growth by Helicobacter pylori: Evidence for a major role of apoptosis. Gastroenterology. (1997) 113:1836-47. doi: 10.1016/S0016-5085(97)70003-9

30. Deeb KK, Trump DL Johnson CS. Vitamin D signaling pathways in cancer: potential for anticancer therapeutics. Cancer. (2007) 7:684-700. doi: $10.1038 / \mathrm{nrc} 2196$

31. Feldman D, Krishnan AV, Swami S, Giovannucci E, Feldman BJ. The role of vitamin D in reducing cancer risk and progression. Nat Rev Cancer. (2014) 14:342-57. doi: 10.1038/nrc3691

32. Wen Y, Da M, Zhang Y, Peng L, Yao J, Duan Y. Alterations in vitamin D signaling pathway in gastric cancer progression: a study of vitamin $\mathrm{D}$ receptor expression in human normal, premalignant, and malignant gastric tissue. Int J Clin Exp Pathol. (2015) 8:13176-84.

33. Khayatzadeh S, Feizi A, Saneei P, Esmaillzadeh A. Vitamin D intake, serum Vitamin D levels, and risk of gastric cancer: a systematic review and metaanalysis. J Res Med Sci. (2015) 20:790-6. doi: 10.4103/1735-1995.168404

34. Kwak JH, Paik JK. Vitamin D status and gastric cancer: a cross-sectional study in Koreans. Nutrients. (2020) 12:2004. doi: 10.3390/nu12072004

35. van Grieken NCT, Meijer GA, Hausen A, Meuwissen SGM, Baak JPA, et al. Increased apoptosis in gastric mucosa adjacent to intestinal metaplasia. J Clin Pathol. (2003) 56:358-62. doi: 10.1136/jcp.56.5.358
36. Charitos IA, D'Agostino D, Topi S, Bottalico L. 40 years of helicobacter pylori: a revolution in biomedical thought. Gastroenterol Insights. (2021) 12:111-35. doi: 10.3390/gastroent 12020011

Conflict of Interest: The authors declare that the research was conducted in the absence of any commercial or financial relationships that could be construed as a potential conflict of interest.

Publisher's Note: All claims expressed in this article are solely those of the authors and do not necessarily represent those of their affiliated organizations, or those of the publisher, the editors and the reviewers. Any product that may be evaluated in this article, or claim that may be made by its manufacturer, is not guaranteed or endorsed by the publisher.

Copyright (C) 2021 Chen, Chang, Hua, Cheng and Lee. This is an open-access article distributed under the terms of the Creative Commons Attribution License (CC BY). The use, distribution or reproduction in other forums is permitted, provided the original author(s) and the copyright owner(s) are credited and that the original publication in this journal is cited, in accordance with accepted academic practice. No use, distribution or reproduction is permitted which does not comply with these terms. 